\title{
Non-diffusive, non-local transport in fluids and plasmas
}

\author{
D. del-Castillo-Negrete \\ Oak Ridge National Laboratory, Oak Ridge, TN, USA
}

Received: 4 August 2010 - Revised: 26 October 2010 - Accepted: 7 November 2010 - Published: 20 December 2010

\begin{abstract}
A review of non-diffusive transport in fluids and plasmas is presented. In the fluid context, nondiffusive chaotic transport by Rossby waves in zonal flows is studied following a Lagrangian approach. In the plasma physics context the problem of interest is test particle transport in pressure-gradient-driven plasma turbulence. In both systems the probability density function (PDF) of particle displacements is strongly non-Gaussian and the statistical moments exhibit super-diffusive anomalous scaling. Fractional diffusion models are proposed and tested in the quantitative description of the non-diffusive Lagrangian statistics of the fluid and plasma problems. Also, fractional diffusion operators are used to construct non-local transport models exhibiting up-hill transport, multivalued flux-gradient relations, fast pulse propagation phenomena, and "tunneling" of perturbations across transport barriers.
\end{abstract}

\section{Introduction}

The widely used advection-diffusion equation rests on the validity of the Fourier-Fick's prescription which in the case of transport of a single scalar, $T$, in a one-dimensional domain states that, $q=-\chi \partial_{x} T+V T$, where $q$ is the flux, $\chi$ is the diffusivity, and $V$ the advection velocity. From the statistical mechanics point of view, this model assumes an underlying Markovian, Gaussian, uncorrelated stochastic process. However, despite the relative success of the diffusion model, there are cases in which this model fails to describe transport, and an alternative description must be used. The goal of this paper is to review some recent results on non-diffusive transport of particular interest to fluids and plasmas. We focus on non-diffusive Lagrangian particle transport and non-local transport of passive scalar fields.

Correspondence to:

D. del-Castillo-Negrete

(delcastillod@ornl.gov)
In the paradigmatic case of the Brownian random walk, the Gaussian statistics of the individual particle displacements, and the lack of correlations and memory effects (Markovian assumption), lead to a Gaussian PDF of the net particle displacement, and to the linear in time scaling for the mean, $M \sim t$, and the variance, $\sigma^{2} \sim t$. Based on these scaling, the transport coefficients are defined as $V=\lim _{t \rightarrow \infty} M(t) / t$ and $\chi=\lim _{t \rightarrow \infty} \sigma^{2}(t) / t$. The signatures of non-diffusive behavior in Lagrangian particle transport include nonGaussian PDFs of particle displacements and anomalous scaling of the moments of the form $M \sim t^{\xi}$ and $\sigma^{2} \sim t^{\gamma}$, with $\xi \neq 1$ and/or $\gamma \neq 1$. When $\gamma>1(\gamma<1)$ transport is superdiffusive (sub-diffusive), see for example Bouchaud (1990). In either case, the diffusion model cannot be applied because the effective diffusivity $\chi$ is either $\infty$ or zero.

The study of non-diffusive Lagrangian particle transport presented here focuses on two systems of interest to geophysical fluid dynamics and plasma physics. In the geophysical context we consider transport in quasigeostrophic zonal flows. Quasigeostrophic flows are 2-D, rapidly rotating flows in which there is a gradient in the Coriolis force. These flows are relevant in the study of mesoscale dynamics in the atmosphere and the oceans, see for example Pedlosky (1987). Zonal shear flows occur naturally in nature; two well-known examples are the Gulf Stream and the polar night jet above Antarctica. Barotropic perturbations of these flows give rise to low frequency instabilities known as Rossby waves that have a key influence on the dynamics and transport. Following del-CastilloNegrete and Morrison (1993), del-Castillo-Negrete (1998) we study chaotic transport by Rossby waves in zonal shear flows. In the plasma physics context we consider non-diffusive transport in pressure-gradient-driven plasma turbulence. This system is of relevance to the understanding of magnetically confined fusion plasmas. In this case, the Lagrangian particle dynamics corresponds to the motion of test particles in the presence of an external fixed magnetic field and a fluctuating turbulent electrostatic potential. In the fluid and the plasma physics problems, we present numerical

Published by Copernicus Publications on behalf of the European Geosciences Union and the American Geophysical Union. 
evidence of non-diffusive transport. In particular, in both cases, the PDFs of particle displacements are strongly nonGaussian and the variance exhibits anomalous scaling of the super-diffusive type.

As mentioned before, when the statistical moments exhibit anomalous scaling, the advection-diffusion model can not be applied and alternative models must be used. In this paper we review the use of fractional derivatives to construct such alternative models. Fractional derivatives are integro-differential operators that generalize the concept of derivatives of order $n$, to fractional orders (Samko et al., 1993; Podlubny, 1999). Although the origins of fractional calculus go back to the origins of regular calculus, the use of fractional derivatives in the mathematical modeling of transport is relative recent. We present a brief review of this formalism in connection with the continuous time random walk (CTRW) model. The CTRW generalizes the Brownian random walk by incorporating non-Gaussian jump PDFs and non-Markovian waiting time PDFs Montroll and Weiss (1965); Montroll and Shlesinger (1984); Metzler and Klafter (2000). Following this, we construct effective macroscopic fractional diffusion models of the PDFs of particle displacements del-Castillo-Negrete et al. (2004, 2005). A comparison is presented between the analytical solutions of the fractional models and the numerical results obtained from the Lagrangian statistics for the fluid and plasma problems mentioned above.

The use of fractional derivatives in transport modeling is closely related to the problem of nonlocal transport. By nonlocal we mean that the flux of the transported scalar at a point depends on the gradient of the scalar throughout the entire domain. The generic mathematical structure of the nonlocal flux is $q=-\chi \int \mathcal{K}(x-y) \partial_{y} T d y$, where the function $\mathcal{K}$ measures the degree of nonlocality. The "width" of this function depends on the strength of the nonlocality, and in the limit when $\mathcal{K}$ is a Dirac delta function, the flux reduces to the local Fourier-Fick's prescription. Motivated by the successful use of fractional derivatives to model non-diffusive Lagrangian transport, we discuss the use of these operators to construct non-local model of passive scalar transport. Following del-Castillo-Negrete (2006); del-Castillo-Negrete et al. (2008), we present numerical results illustrating important non-local transport phenomenology including: up-hill transport, multivalued flux-gradient relations, fast pulse propagation phenomena, and "tunneling" of perturbations across transport barriers.

The rest of this paper is organized as follows. Section 2 discusses non-diffusive chaotic transport by Rossby waves in zonal flows. Non-diffusive turbulence transport in plasmas is studied in Sect. 3. Section 4 presents a brief review of fractional diffusion. The applications of fractional diffusion to model the PDFs of particle displacements in the Rossby waves and the plasma problems are discussed in Sect. 5. Non-local transport is studied in Sect. 6, and Sect. 7 presents the conclusions.

\section{Non-diffusive chaotic transport by Rossby waves in zonal flows}

In this section we study non-diffusive chaotic transport by Rossby waves in zonal shear flows. Since the flow is 2-D and incompressible, the flow velocity can be written as $\mathbf{v}=\left(-\partial_{y} \Psi, \partial_{x} \Psi\right)$ where $\Psi(x, y, t)$ is the streamfunction. In this case, the Lagrangian trajectories of individual tracers, $d \mathbf{r} / d t=\mathbf{v}$, are obtained from the solution of the Hamiltonian system,

$\frac{d x}{d t}=-\frac{\partial \Psi}{\partial y} \quad \frac{d y}{d t}=\frac{\partial \Psi}{\partial x}$.

where $\Psi$ plays the role of the Hamiltonian and the $\mathbf{r}=(x, y)$ spatial coordinates play the role of canonically conjugate phase space coordinates. Hamiltonian systems of the form in Eq. (1) are always integrable when $\Psi$ does not depend on time. However, when $\Psi$ depends explicitly on time, the system can be non-integrable and individual trajectories can be chaotic, see for example Tabor (1989). The main goal of the study of chaotic transport is to understand the global transport properties of tracers in this case, see for example Ottino (1989). Problems of particular interest to geophysical flows include the study of the formation and destruction of transport barriers (del-Castillo-Negrete and Morrison, 1993), and the study of the Lagrangian statistics (del-Castillo-Negrete, 1998). Here we focus on the second problem.

To construct a model for the streamfunction $\Psi(x, y, t)$ we have to consider the dynamics of the system. In the case of quasigeostrophic flows, $\Psi(x, y, t)$ is obtained from the potential vorticity conservation law

$\frac{\partial q}{\partial t}+(\mathbf{v} \cdot \nabla) q=0$,

where according to the $\beta$-plane approximation, $q=\nabla^{2} \Psi+$ $\beta y$. We have adopted a right-handed Cartesian coordinate system with $z$ pointing in the direction of the rotation of the system and $y$ in the direction of the Coriolis force gradient. That is, $y$ points in the "northward" direction and $x$ is a periodic coordinate in the "eastward" direction.

To simplify the solution of the non-linear Eq. (2) we assume a streamfunction of the form

$\Psi=\Psi_{0}(x, y)+\Psi_{1}(x, y, t)$,

where $\Psi_{0}$, is the superposition of a zonal shear flow with dependence $u_{0}(y)=\tanh y$, and a regular neutral mode in its co-moving reference frame,

$\Psi_{0}=-\ln (\cosh y)+\epsilon_{1} \phi_{1}(y) \cos \left(k_{1} x\right)+c_{1} y$.

The function $\Psi_{1}$ is a time dependent perturbation of the form

$\Psi_{1}=\epsilon_{2} \phi_{1}(y) \cos \left(k_{1} x-\omega t\right)$, 
where $\epsilon_{1}$ and $\epsilon_{2}$ are free parameters determining the amplitude of the linear Rossby waves, and $\omega$ is the frequency of the perturbation. The eigenfunction $\phi_{1}$,

$$
\phi_{1}=[1+\tanh y]^{\left(1-c_{1}\right) / 2}[1-\tanh y]^{\left(1+c_{1}\right) / 2},
$$

is obtained from the solution of the linear eigenvalue problem of the quasigeostrophic equation and $\left(k_{1}, c_{1}\right)$ are obtained from the corresponding dispersion relation for neutral (zero growth rate) modes (del-Castillo-Negrete, 1998).

When $\epsilon_{2}=0$ the streamfunction is time independent and the solution of Eq. (1) can be reduced to a quadrature. In this case the Lagrangian dynamics is integrable and the orbits of the tracers can be classified in two types: (i) trapped orbits that encircle the vortices and (ii) untrapped orbits that move freely in the East-West direction following the zonal shear flow. These two types of orbits are separated by the separatrix that joints the hyperbolic stagnation points of the flow. When $\epsilon_{2} \neq 0$, the system ceases to be integrable. In particular, as shown in Fig. 1, the perturbation breaks the separatrix and creates a stochastic layer where tracers alternate chaotically between following the zonal flow and being trapped inside the vortices.

To characterize transport in the chaotic regime we follow a statistical approach. The most basic quantity is the probability density function (PDF) of particle displacements. Transport in the "north-south" direction is trivial since particle orbits in the y-direction are bounded by the zonal flows. Therefore, we focus on transport in the "east-west" direction, i.e. along the zonal flow. Given an ensemble of initial conditions, $\left\{\left(x_{i}, y_{i}\right)\right\}$ with $i=1,2, \ldots N_{\mathrm{p}}$ we compute the PDF of particle displacements, $P(\delta x, t)$ where $\delta x_{i}(t)=$ $x_{i}(t)-x_{i}(0)$. By definition, at $t=0$ the PDF is a Dirac delta function, $P(\delta x, t=0)=\delta(\delta x)$. As $t$ increases, the PDF widens and might drift to one side or the other. Note that, although $\delta x$ is a periodic function in the annular domain shown in Fig. 1, to compute the statistics we treat $\delta x$ as variable defined on the $(-\infty, \infty)$ domain.

To study the self-similar evolution of the PDF we introduce the scaling variable

$\eta=\langle\delta x-\langle\delta x\rangle\rangle t^{-\gamma / 2}$.

Figure 2 shows the rescaled PDF, $t^{\gamma / 2} P$, as function of $\eta$. The observation that the rescaled PDFs collapse for successive times leads support to the assumption that, at large times, $P$ converges to a self-similar distribution of the form

$P^{*}(x, t)=t^{-\gamma / 2} f(\eta)$,

where $f$ is a scaling function, and $\gamma$ is the scaling exponent. The scaling in Eq. (8) implies the following scaling of the moments

$$
\left\langle X^{n}\right\rangle \sim t^{n \gamma / 2},
$$

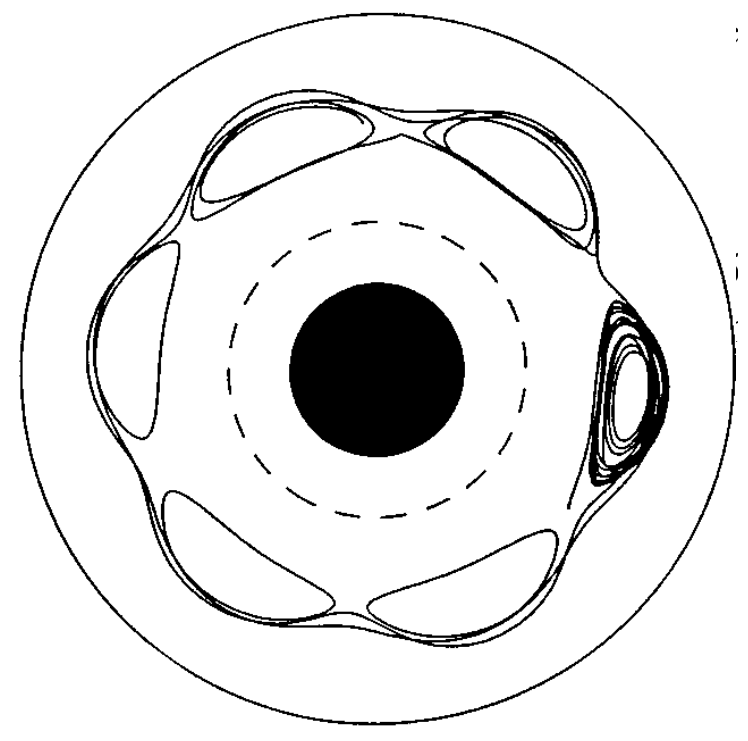

Fig. 1. Chaotic transport by Rossby waves in the quasigeostrophic zonal flow in Eqs. (1) and (3). In the presence of two or more Rossby waves, the trajectories of passive tracers are typically chaotic. In particular, as shown in the figure, tracers alternate in a seemly random way between being trapped in the vortices and moving freely along the "east-west", $\mathrm{x}$ angular direction, following the shear flow flanking the vortices.

where $X=\delta x-\langle\delta x\rangle$. Equation (8) also implies

$P^{*}(X, t)=\lambda^{\gamma / 2} P^{*}\left(\lambda^{\gamma / 2} X, \lambda t\right)$,

where $\lambda$ is a free parameter. According to this relation, up to a scale factor, the limit distribution, $P^{*}$, is invariant under the space-time renormalization operation $(X, t) \rightarrow\left(\lambda^{\gamma / 2} X, \lambda t\right)$. That is, the PDF at a later time can be obtained from a rescaling of the PDF at an early time.

In the diffusive case, $P^{*}$ is a Gaussian, $\gamma=1$, and Eq. (8) corresponds to the similarity solution of the advectiondiffusion equation. However, in the numerical results shown in Fig. 2, transport is non-diffusive because $\gamma \neq 1$ and the scaling function is not a Gaussian. In particular, the tails of the PDFs exhibit a decay significantly slower than Gaussian and a strong asymmetry. Because, $\gamma>1$, it is concluded that "East-West", azimuthal chaotic transport by Rossby waves in zonal flows is super-diffusive. For further details on the statistics and a dynamical explanation of the dependence of the asymmetry of the PDF on the perturbation frequency $\omega$ see del-Castillo-Negrete (1998). This reference also discusses the comparison of the model presented here with experimental results on transport in rapidly rotating fluids (Solomon et al., 1993).

It is interesting to mention that there is a very close analogy between the dynamics of Rossby waves in rapidly rotating neutral fluids in the quasigeostrophic approximation 


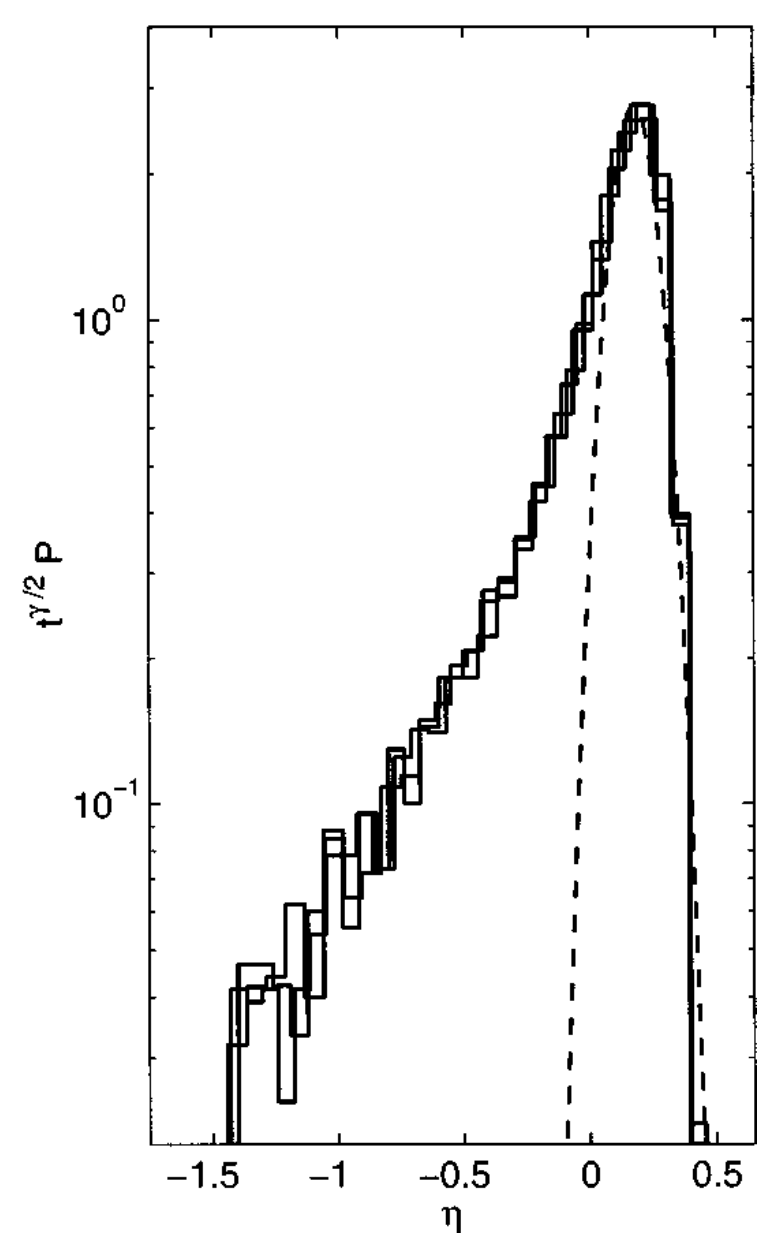

Fig. 2. Rescaled probability distribution function (PDF), $t^{\gamma / 2} P$, of passive tracers displacements, $\delta x(t)=x(t)-x(t=0)$, as function of the similarity variable, $\eta=(\delta x-\langle\delta x\rangle) / t^{\gamma / 2}$ with $\gamma=1.9$. The dynamics corresponds to the quasigeostrophic model in Eqs. (1) and (3). The plot shows the PDF at $t=800,900$ and 1000. Consistent with the self-similar scaling in Eq. (8), the PDFs at successive times collapse. The anomalously large displacements induced by the zonal flow (see Fig. 1) result in the strong departure of the $\eta<0$ tail from the Gaussian fit (dashed line). The value $\gamma>1$ indicates super-diffusive transport.

and drift-waves in magnetized plasmas, see for example Petviashvili and Pokhotelov (1992), Horton and Hasegawa (1994), Horton and Ichikawa (1996). In this analogy, the role of the rapid rotation is played by the strong magnetic field, the fluid streamfunction corresponds to the electrostatic potential, the fluid vorticity to the plasma density, and the gradient in the Coriolis force corresponds to the plasma density background gradient. Based on this analogy, as discussed in del-Castillo-Negrete (2000), the results presented here are directly applicable to the study of non-diffusive chaotic transport by drift waves in magnetized plasmas.

\section{Non-diffusive turbulent transport in plasmas}

In the example discussed in the previous section, transport resulted from chaotic advection. That is, from the chaotic dynamics of the deterministic equations describing the particle orbits. In particular, the streamfunction $\Psi$ is a deterministic differentiable function. In the case of turbulent transport the situation is different since the flow velocity advecting the tracers is a nondeterministic, random function. Nevertheless, turbulent systems can also exhibit non-diffusive transport of passive tracers. In this section we present an example in the context of plasma physics.

As in the previous section, we follow a Lagrangian approach and consider the statistics of a large ensemble of tracer particles. In the plasma, the particle motion responds to the combined effect of a turbulent electric field, $\tilde{\mathbf{E}}=-\nabla \tilde{\Phi}$, and a fixed external magnetic field, $\mathbf{B}_{0}$. The equation of motion of the tracers are obtained from Newton's law with the Lorentz force. However, in the guiding center approximation, see for example Nicholson (1983), the equations can be simplified as the first order system

$\frac{d \mathbf{r}}{d t}=\frac{1}{B_{0}^{2}} \nabla \tilde{\Phi} \times \mathbf{B}_{0}$,

where $\mathbf{r}=(x, y)$ denotes the position of the particle in the 2-D plane perpendicular to the magnetic field. This system has also a Hamiltonian structure with the potential, $\tilde{\Phi}$, playing the role of Hamiltonian.

The fluctuating plasma electrostatic potential, $\tilde{\Phi}$, is obtained from the solution of the turbulence model. Here, following del-Castillo-Negrete et al. (2004, 2005), we consider pressure-gradient-driven turbulence in cylindrical geometry. The underlying instability of this type of turbulence is the resistive interchange mode, driven by the pressure gradient. This instability is the analogue of the Rayleigh-Taylor instability responsible for the gravitydriven overturning of high density fluid laying above a low density fluid. In magnetically confined plasmas, the role of gravity is played by the magnetic field lines curvature. The turbulence model (Carreras, et al., 1987) is based on an electrostatic approximation of the reduced resistive magneto hydrodynamic equations,

$$
\begin{aligned}
& \frac{d}{d t} \nabla_{\perp}^{2} \tilde{\Phi}=-\frac{1}{\eta m_{\mathrm{i}} n_{0} R_{0}} \nabla_{\|}^{2} \tilde{\Phi}+\frac{B_{0}}{m_{\mathrm{i}} n_{0}} \frac{1}{r_{\mathrm{c}}} \frac{1}{r} \frac{\partial \tilde{p}}{\partial \theta}+\mu \nabla_{\perp}^{4} \tilde{\Phi}, \\
& \frac{d}{d t} \tilde{p}=\frac{\partial\langle p\rangle}{\partial r} \frac{1}{r} \frac{\partial \tilde{\Phi}}{\partial \theta}+\chi_{\perp} \nabla_{\perp}^{2} \tilde{p}+\chi_{\|} \nabla_{\|}^{2} \tilde{p},
\end{aligned}
$$

where $\tilde{\Phi}$ is the electrostatic potential, $\tilde{p}$ the pressure, and $d / d t=\partial_{\tau}+\tilde{\mathbf{V}} \cdot \nabla$. The instability driver is the flux-surface averaged pressure gradient, $\partial\langle p\rangle / \partial r$, determined according to

$$
\frac{\partial\langle p\rangle}{\partial \tau}+\frac{1}{r} \frac{\partial}{\partial r} r\left\langle\tilde{V}_{r} \tilde{p}\right\rangle=S_{0}+D \frac{1}{r} \frac{\partial}{\partial r}\left(r \frac{\partial\langle p\rangle}{\partial r}\right) .
$$


The tildes indicate fluctuating quantities (in space and time), and the angular brackets, \langle\rangle , denote flux surface averaging over a cylinder at a fixed radius. The equilibrium density is $n_{0}$, the ion mass is $m_{\mathrm{i}}$, the averaged radius of curvature of the magnetic field lines is $r_{\mathrm{c}}$, and the resistivity is $\eta$. The sub indices " $\perp$ " and " $\|$ " denote the direction perpendicular and parallel to the magnetic field, respectively. The function $S_{0}$ represents a source of particles and heat which we model using a parabolic profile, $S_{0}=$ $\bar{S}_{0}\left[1-(r / a)^{2}\right]$. Figure 3 shows a snapshot in time of the fluctuating electrostatic potential $\tilde{\Phi}$ obtained form the solution of Eqs. (12)-(14).

Having computed $\tilde{\Phi}$, the next step is to integrate Eq. (11) to obtain the orbits of the tracers. The initial condition consists of $25 \times 10^{3}$ particles with random initial positions in $\theta$ and $z$, and radial position $r=0.5 a$. By definition, at $t=0$, the PDF, $P$, of radial particle displacements, $x=$ $[r(t)-r(0)] / a$, is a Dirac delta function. As time advances the $P(x, t)$, spreads and develop slowly decaying, "fat" tails. Figure 4 shows the long-time behavior of the PDF as function of the similarity variable $x / t^{\nu}$. The strong nonGaussianity of $P$ is evident. Like in the previous fluid example case, transport is super-diffusive because $v>1 / 2$. Evidence of non-diffusive transport has also been observed in other plasma systems including gyrokinetic simulations of ion temperature gradient (ITG) turbulence (Sanchez et al., 2008).

\section{Fractional diffusion models of non-diffusive transport}

One of the main goals of transport modeling is to construct effective macroscopic transport equations that reproduce experimentally or numerically observed phenomena. For example, in the fluid and the plasma transport problems discussed in the previous two sections, the goal is to construct a transport equation that describes the observed spatio-temporal evolution of the PDF, $P$, of particle displacements.

When transport is diffusive, a simple solution to this problem is provided by the advection-diffusion equation

$\partial_{t} P+V \partial_{x} P=\partial_{x}\left(\chi \partial_{x} P\right)$,

where the advection velocity and diffusivity are obtained from the asymptotic behavior the statistical moments

$V=\lim _{t \rightarrow \infty} \frac{\langle x(t)\rangle}{t}, \quad \chi=\lim _{t \rightarrow \infty} \frac{\left\langle x^{2}(t)\right\rangle}{2 t}$,

of the particle's displacements, $x$. However, this approach fails in the case of non-diffusive transport. In particular, according to the scaling in Eq. (9) when there is superdiffusion, $\chi \rightarrow \infty$. Moreover, as it is well-known, the Green's function of Eq. (15) in an unbounded domain, is a translated Gaussian and this significantly limits the range

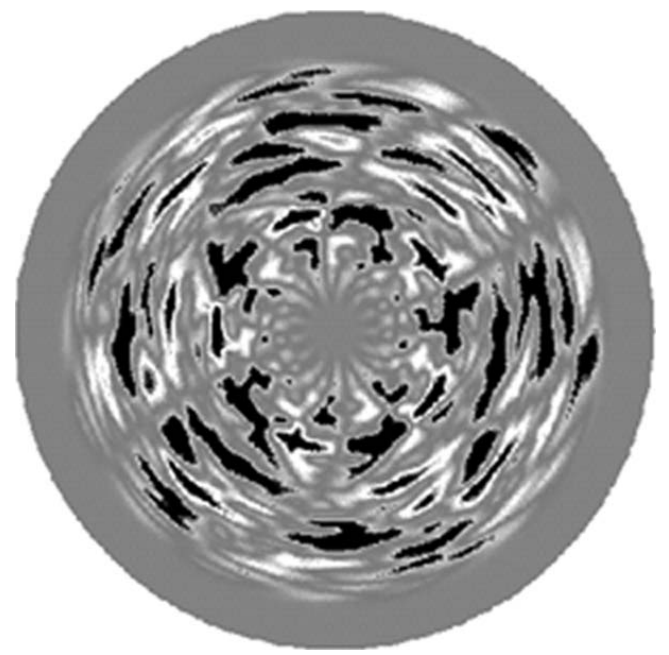

Fig. 3. Fluctuating electrostatic potential $\tilde{\Phi}$ at a fixed time obtained from the numerical integration of the plasma turbulence model in Eqs. (12)-(14). The dark (light) coherent patches denote rotating (counter rotating) $\mathbf{E} \times \mathbf{B}$ eddies. The trapping effects of these eddies along with intermittent large radial displacements caused by avalanche-like plasma relaxation events, give rise to non-diffusive transport and to the non-Gaussian PDF in Fig. 4 (del-CastilloNegrete et al., 2004, 2005).

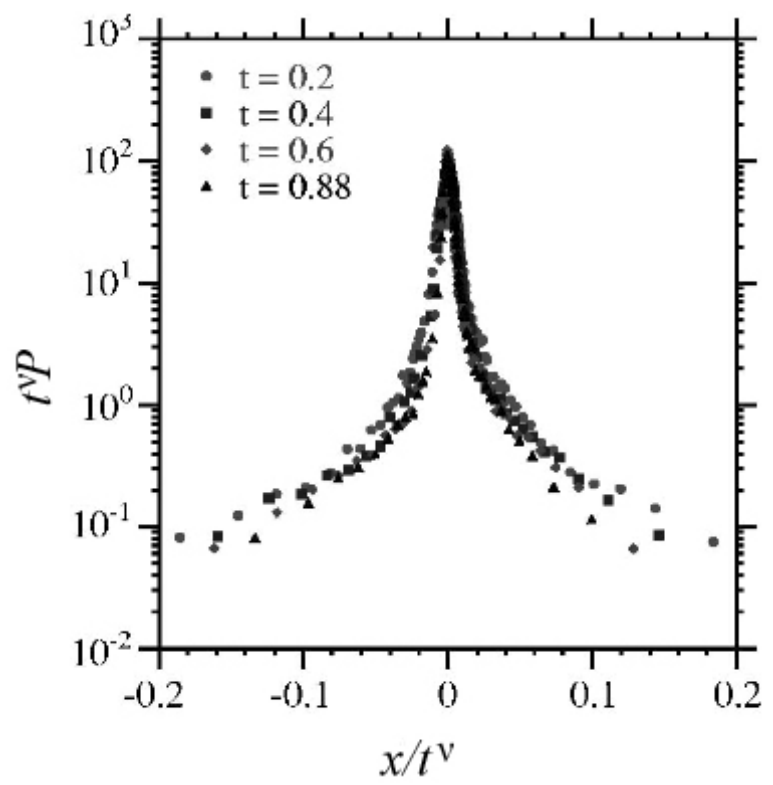

Fig. 4. Rescaled probability distribution functions (PDFs), $t^{\nu} P$, of passive tracers radial displacements, $x(t)$, as function of the similarity variable, $x / t^{v}$ with $v=2 / 3$. The dynamics correspond to the pressure-gradient-driven plasma turbulence model in Eqs. (12)-(14). The plot shows the PDF at $t=0.2,0.4,0.6$ and 0.88. Like in the fluid dynamic case in Fig. 2, the collapse of the PDFs at successive times indicates a self-similar scaling of the form in Eq. (8). In this case, the non-diffusive transport manifest in the slowly decaying non-Gaussian tails of the PDF. The value $v>1 / 2$ indicates super-diffusive transport (del-Castillo-Negrete et al., 2004, 2005). 
of PDFs that this model can describe. In particular, PDFs with $\gamma \neq 1$ scaling and/or with slowly decaying tails, like those obtained in the examples discussed before (Figs. 2 and 4), cannot be modeled using a simple advection-diffusion equation.

From the statistical mechanics point of view, the advection-diffusion model assumes an underlying Markovian, Gaussian stochastic process with a drift, i.e. a biased Brownian random walk, see for example Paul and Baschnagel (1999). However, the description of transport in the presence of coherent structures requires the use of random walk models that incorporate more general stochastic processes. In particular, in the fluid problem discussed in Sect. 2, the trapping effect of the vortices gives rise to non-Markovian effects, and the zonal shear flows give rise to non-Gaussian particle displacements. In the plasma physics problem discussed in Sect. 3, the non-Markovian effects are due to the trapping in electrostatic eddies, and the non-Gaussian particle displacements result from avalanchelike radial relaxation events.

The Continuous Time Random Walk (CTRW) model (Montroll and Weiss, 1965; Montroll and Shlesinger, 1984; Metzler and Klafter, 2000) provides an elegant powerful framework to incorporate this type of effects. The CTRW generalizes the Brownian walk in two ways. First, contrary to the Brownian random walk where particles are assumed to jump at discrete fixed time intervals, the CTRW model allows the possibility of incorporating a waiting time probability distribution, $\psi(t)$. In addition, the CTRW model allows the possibility of using non-Gaussian jump distribution functions, $\eta(x)$, with divergent moments to account for long displacements known as Lévy flights. Given $\psi$ and $\eta$, the probability of finding a tracer at position $x$ and time $t$ is determined by the Montroll-Weiss master equation

$$
\begin{aligned}
\partial_{t} P= & \int_{0}^{t} d t^{\prime} \phi\left(t-t^{\prime}\right) \int_{-\infty}^{\infty} d x^{\prime} \\
& {\left[\eta\left(x-x^{\prime}\right) P\left(x^{\prime}, t^{\prime}\right)-\eta\left(x-x^{\prime}\right) P\left(x, t^{\prime}\right)\right], }
\end{aligned}
$$

The spatial integral on the right-hand-side represents the gain-loss balance for $P$ at $x$. In particular, the first term inside the square bracket gives the increase of $P$ due to particles moving to $x$ while the second term describes the decrease of $P$ due to particles moving away from $x$. The time integral accounts for memory effects weighted by the function $\phi(t)$. In Fourier-Laplace variables,

$$
\begin{aligned}
& \mathcal{F}[\eta]=\hat{\eta}(k)=\int_{-\infty}^{\infty} e^{\mathrm{ikx}} \eta(x) d x, \\
& \mathcal{L}[\phi]=\tilde{\phi}(s)=\int_{0}^{\infty} e^{-\mathrm{st}} \phi(t) d t,
\end{aligned}
$$

Eq. (17) takes the form

$$
\hat{\tilde{P}}(k, s)=\frac{1-\tilde{\psi}(s)}{s} \frac{1}{1-\tilde{\psi}(s) \hat{\eta}(k)} .
$$

where the relation between the waiting time PDF and the memory function is $\tilde{\phi}=s \tilde{\psi} /(1-\tilde{\psi})$.

The Montroll-Weiss master Eq. (17) can be used directly to model non-diffusive transport, see for example van Milligen et al. (2004); Spizzo et al. (2009). However, this description carries in a sense too much information concerning the details of the underlying stochastic process that might be irrelevant in the long-time, large-scale description of transport. This motivates the derivation of a macroscopic transport equation from Eq. (20) valid in the time asymptotic $(s \rightarrow 0)$ long-wavelength $(k \rightarrow 0)$ "continuum" limit (Saichev and Zaslavsky, 1997; Metzler and Klafter, 2000; Scalas et al., 2004). A key aspect of this limit is that only the asymptotic behavior, i.e., the tails of the $\eta$ and $\psi$ PDFs matter. This is a significant advantage over the use of the kinetic master equation that requires the detailed knowledge of these functions.

As expected, in the Markovian-Gaussian case

$\psi(t)=\mu e^{-\mu t}, \quad \eta(x)=\frac{1}{\sqrt{2 \pi} \sigma} e^{-x^{2} /\left(2 \sigma^{2}\right)}$,

where $\langle t\rangle=1 / \mu$ is the characteristic waiting time and $\sigma^{2}=$ $\left\langle x^{2}\right\rangle$ is the characteristic mean square jump, the fluid limit of the master equation Eq. (20) leads to the standard diffusion equation in (15). However, the situation is quite different in the case of algebraic decaying PDFs of the form

$\psi \sim t^{-(\beta+1)}, \quad \eta \sim|x|^{-(\alpha+1)}$,

where for simplicity we have assumed that $\eta$ is symmetric. In this case, for $0<\beta<1,\langle t\rangle$ diverges, and there is no characteristic waiting time. Similarity, for $\alpha<2,\left\langle x^{2}\right\rangle$ diverges, indicating a lack of characteristic transport scale. The use of this type of algebraic decaying PDFs is motivated by the significant probability of very large trapping events and very large spatial displacements, as it is the case in the examples discussed in Sects. 2 and 3. From the asymptotic behavior in Eq. (22) it follows that for small $s$ and $k$,

$\tilde{\psi}(s) \approx 1-s^{\beta}+\ldots, \quad \hat{\eta}(k) \approx 1-|k|^{\alpha}+\ldots$

Substituting Eq. (23) into Eq. (20) we get to leading order

$s^{\beta} \hat{\tilde{P}}(k, s)-s^{\beta-1}=-\chi|k|^{\alpha} \hat{\tilde{P}}(k, s)$.

To obtain the macroscopic transport equation we need to invert the Fourier-Laplace transforms in Eq. (24). This can be formally done by writing

${ }_{0}^{c} D_{t}^{\beta} P=\chi D_{|x|}^{\alpha} P$,

where the operators in Eq. (25) are defined according to

$\mathcal{L}\left[{ }_{0}^{c} D_{t}^{\beta} P\right]=s^{\beta} \tilde{P}(x, s)-s^{\beta-1} \delta(x)$,

$\mathcal{F}\left[D_{|x|}^{\alpha} P\right]=-|k|^{\alpha} \hat{P}(k, t)$, 
for $0<\beta<1$. Equations (26) and (27) are the natural generalizations of the Laplace transform of a time derivative and the Fourier transform of a spatial derivative. This motivates the formal identification of the operator ${ }_{0}^{c} D_{t}^{\beta}$ as a "fractional time derivative" for $0<\beta<1$, and the operator $D_{|x|}^{\alpha}$ as a "fractional space derivative" for $1<\alpha<2$. As expected, for $\alpha$ or $\beta$ integers, the regular derivatives are recovered.

The previous discussion assumed a symmetric jump stochastic process, $\eta(x)=\eta(-x)$. It can be shown that in the general case the transport equation is

${ }_{0}^{c} D_{t}^{\beta} P=\chi\left[l_{-\infty} D_{x}^{\alpha}+r_{x} D_{\infty}^{\alpha}\right] P$,

where the operators on the right hand side are the left and right Riemann-Liouville fractional derivatives of order $\alpha$ (Samko et al., 1993; Podlubny, 1999)

${ }_{a} D_{x}^{\alpha} f=\frac{1}{\Gamma(m-\alpha)} \frac{\partial^{m}}{\partial x^{m}} \int_{a}^{x} \frac{f(y)}{(x-y)^{\alpha+1-m}} d y$,

${ }_{x} D_{b}^{\alpha} f=\frac{(-1)^{m}}{\Gamma(m-\alpha)} \frac{\partial^{m}}{\partial x^{m}} \int_{x}^{b} \frac{f(y)}{(y-x)^{\alpha+1-m}} d y$,

where $m$ is a positive integer such that $m-1 \leq \alpha<m$. In this general formulation, the asymmetry of the underlying stochastic process manifests on the parameters $l$ and $r$,

$l=-\frac{(1-\theta)}{2 \cos (\alpha \pi / 2)}, \quad r=-\frac{(1+\theta)}{2 \cos (\alpha \pi / 2)}$,

that control the relative weight of the left and right fractional derivatives, where $-1 \leq \theta \leq 1$. In the symmetric case, $\theta=0, D_{|x|}^{\alpha}=\frac{-1}{2 \cos (\pi \alpha / 2)}\left[-\infty D_{x}^{\alpha}+{ }_{x} D_{\infty}^{\alpha}\right]$ which corresponds to the operator defined in Fourier space in Eq. (27). In the time domain, the fractional derivative operator in time, ${ }_{0}^{c} D_{t}^{\beta}$, introduced in Eq. (26) become an integro-differential operator of the form

${ }_{0}^{c} D_{t}^{\beta} P=\frac{1}{\Gamma(1-\beta)} \int_{0}^{t} \frac{\partial_{t^{\prime}} P}{\left(t-t^{\prime}\right)^{\beta}} d t^{\prime}$,

where $0<\beta<1$. For a derivation of fractional diffusion models that incorporate more general stochastic processes, including the physically important case of truncated Lévy statistics, see Cartea and del-Castillo-Negrete (2007). For a derivation of fractional diffusion models using quasi-linear type renormalization techniques see Sanchez et al. (2006).

\section{Applications of Fractional diffusion models}

The goal of this section is to use the fractional diffusion equation to model the non-diffusive transport of tracers discussed in Sects. 2 and 3. In particular, we show that the numerically obtained PDFs of the particle displacements in Figs. 2 and 4 can be obtained as solutions of effective macroscopic fractional diffusion equations.
The solution of the initial value problem of Eq. (28) with $P(x, t=0)=P_{0}(x)$ is

$P(x, t)=\int_{-\infty}^{\infty} P_{0}\left(x^{\prime}\right) G\left(x-x^{\prime}, t\right) d x^{\prime}$,

where the Green's function (propagator) $G$ is the solution of the initial value problem $G(x, t=0)=\delta(x)$ with $\delta(x)$ the Dirac delta function. Using Eqs. (26) and (27), the FourierLaplace transform of Eq. (28) leads to the solution

$\hat{\tilde{G}}=\frac{s^{\beta-1}}{s^{\beta}-\Lambda(k)}$,

where

$\Lambda=\chi\left[l(-\mathrm{ik})^{\alpha}+\mathrm{r}(\mathrm{ik})^{\alpha}\right]$,

for $\alpha \neq 1$. Introducing the Mittag-Leffler function, see for example Podlubny (1999),

$E_{\beta}(z)=\sum_{n=0}^{\infty} \frac{z^{n}}{\Gamma(\beta n+1)}, \quad \mathcal{L}\left[E_{\beta}\left(c t^{\beta}\right)\right]=\frac{s^{\beta-1}}{s^{\beta}-c}$,

the inversion of the Fourier-Laplace transform in Eq. (34) gives

$G(x, t)=t^{-\beta / \alpha} K(\eta)$,

$K(\eta)=\frac{1}{2 \pi} \int_{-\infty}^{\infty} e^{-i \eta k} E_{\beta}[\Lambda(k)] d k$,

where

$\eta=x\left(\chi^{1 / \beta} t\right)^{-\beta / \alpha}$

is the similarity variable.

Further details of the solution of the initial value problem and useful asymptotic and convergent expansions of the Green's function can be found in Metzler and Klafter (2000); Saichev and Zaslavsky (1997); Mainardi et al. (2001).

Of particular interest is the asymptotic behavior in $x$, for a fixed $t=t_{0}$,

$G\left(x, t_{0}\right) \sim x^{-(1+\alpha)}, \quad x \gg\left(\chi_{f}^{1 / \beta} t_{0}\right)^{\beta / \alpha}$.

and the small $t$ and large $t$ scaling at fixed $x=x_{0}$,

$G\left(x_{0}, t\right) \sim \begin{cases}t^{\beta} & \text { for } t \ll\left(\chi_{f}^{-1} x_{0}^{\alpha}\right)^{1 / \beta} \\ t^{-\beta} & \text { for } t \gg\left(\chi_{f}^{-1} x_{0}^{\alpha}\right)^{1 / \beta} .\end{cases}$

From these relations it follows that the order of the fractional derivative in space, $\alpha$, determines the algebraic asymptotic scaling of the propagator in space for a fixed time, and the order of the fractional derivative in time, $\beta$, determines the asymptotic algebraic scaling of the propagator in time for a fixed $x$. These two properties provide a useful guide to construct fractional models given the spatio-temporal 


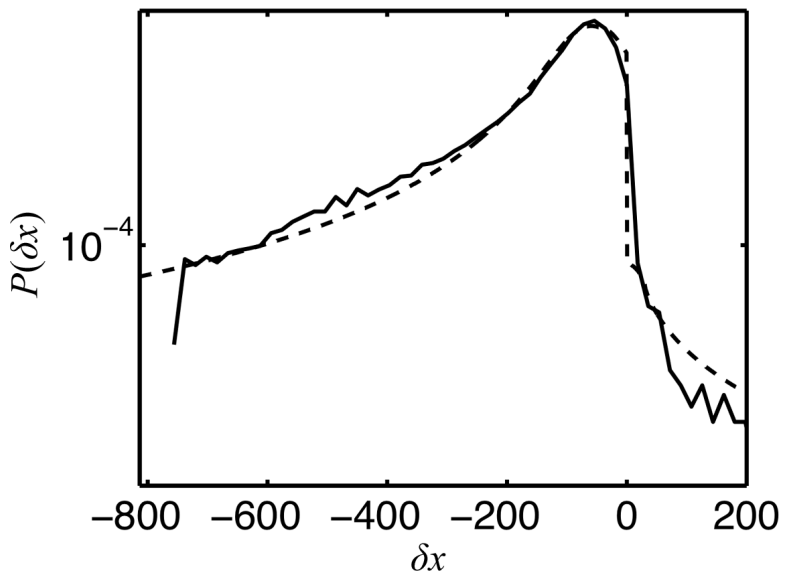

Fig. 5. Comparison between the PDF of particle displacements, $\delta x$, in the quasigeostrophic zonal with Rossby waves(solid line), and the PDF obtained from the solution of the fractional diffusion model in Eq. (28) with $\alpha=\beta=0.9$, and $\theta=1$.

asymptotic scaling properties of the PDF. Using Eq. (37), the moments in the fractional model are given by

$\left\langle x^{n}\right\rangle=\int x^{n} P(x, t) d x \sim t^{n \beta / \alpha} \int \eta^{n} K(\eta) d \eta$,

that implies the anomalous diffusion scaling

$\left\langle x^{2}\right\rangle \sim t^{\gamma}, \quad \gamma=2 \beta / \alpha$.

According to Fig. 2, the scaling exponent of the PDF of particle displacements in chaotic transport by Rossby waves is $\gamma \sim 1.9$. As expected, this value is also consistent with the scaling of the second moment computed directly from the Lagrangian statistic of displacements. Based on this, in the construction of the fractional model we assume $\gamma=2$, which according to Eq. (43) implies $\alpha=\beta$. This special case corresponds to the neutral fractional diffusion equation, for which $G$ in Eq. (37) is (Mainardi et al., 2001):

$G(x, t)=\frac{t^{-1}}{\pi} \frac{\sin [\pi(\alpha-\zeta) / 2] \eta^{\alpha-1}}{1+2 \eta^{\alpha} \cos [\pi(\alpha-\zeta) / 2]+\eta^{2 \alpha}}$,

for $\eta>0$ where $\eta=\delta x / t$ is the similarity variable and $\theta=$ $\tan (\pi \zeta / 2) / \tan (\pi \alpha / 2)$. The solution for $\eta<0$ is obtained using the relation $K(-\eta ; \alpha, \zeta)=K(\eta ; \alpha,-\zeta)$. Figure 5 shows a comparison between the fractional diffusion solution in Eqs. (33) and (44) with the PDF obtained in Sect. 2 from the Lagrangian statistics of the quasigeostrophic transport problem.

In the case of turbulent transport in pressure-gradientdriven plasma turbulence, the asymptotic scaling analysis of the PDFs of particle displacements according to Eqs. (40) and (41) and the super-diffusive scaling of the moments in Eq. (42), indicate that $\alpha=3 / 4$ and $\beta=1 / 2$. Figure 6

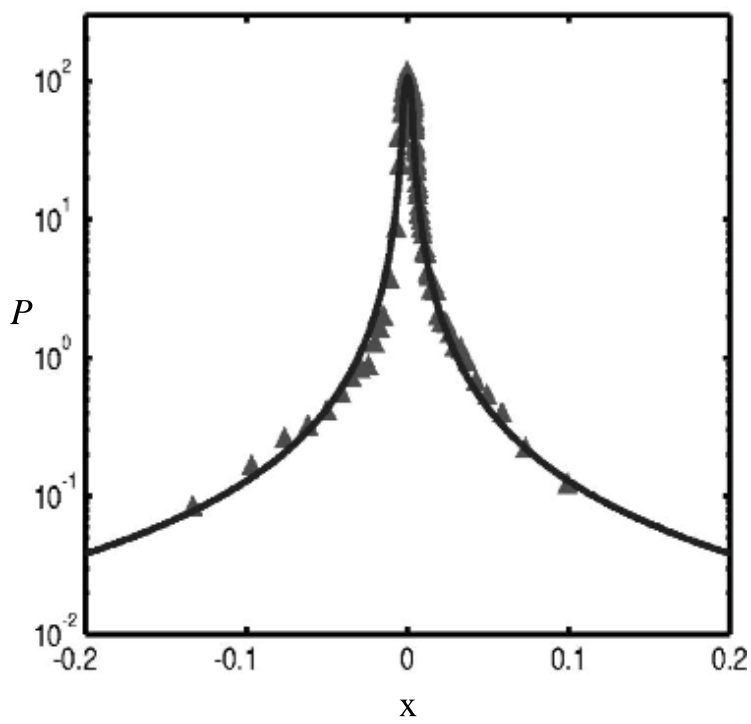

Fig. 6. Comparison between the PDF of particle displacements, $x$, in the resistive, pressure-gradient-driven plasma turbulence model in Eqs. (12)-(14) and Fig. 4 (triangles), and the PDF obtained from the solution of the fractional diffusion model in Eq. (28) with $\alpha=$ $3 / 4, \beta=1 / 2, \theta=0$, and $\chi=0.09$ (del-Castillo-Negrete et al., 2004, 2005).

compares the solution of the fractional diffusion equation for these parameters with the PDF obtained from the direct numerical simulation shown in Fig. 4. Details on the explicit solution of the fractional diffusion equation can be found in del-Castillo-Negrete et al. (2004, 2005). As discussed in Sect. 3, the Lagrangian study of transport in plasmas was based on the guiding-center equations of motion which are an approximation to the dynamics valid in the limit of zero Larmor radius. The role of finite Larmor radius effects on non-diffusive transport, in particular on fractional diffusion was studied in Gustafson et al. (2008).

\section{Non-local transport}

In the previous sections we discussed non-diffusive transport in the context of test particle Lagrangian transport in fluids and plasmas. One of the main goals was to construct macroscopic effective transport models to describe the PDF of particle displacements in chaotic and turbulent flows. It was shown that fractional diffusion operators provide a framework to describe the spatio-temporal evolution of the PDFs. In particular, the long tails of the PDFs as well as the non-Gaussian scaling of the Lagrangian statistics are well captured by fractional diffusion models. Motivated by these results, in this section we discuss the use of fractional diffusion models to describe non-diffusive transport of passive scalars, like temperature, density, pressure or the concentration of a pollutant in flow. 
The starting point is the conservation law

$\partial_{t} T=-\partial_{x} q$,

where $T$ denotes the scalar field transported and $q$ denotes the flux. For simplicity we limit attention to the transport of a single scalar in a 1-D domain. The conservation law (45) has to be complemented with a prescription relating $q$ and $T$. In the case of diffusive-transport this closure is provided by the Fourier-Fick's local prescription

$q=-\chi \partial_{x} T+V T$,

where $\chi$ is diffusion coefficient and $V$ is the advection velocity. Substituting Eq. (46) into Eq. (45) leads to the advection-diffusion model in Eq. (15).

Although the advection-diffusion model has been successfully applied to a wide variety of transport problems, there are cases in which this model fails to describe the dynamics. The examples discussed before showed clear evidence of this in the case of the PDF of Lagrangian particle displacements. Here we explore the role of non-diffusive transport of scalars, like temperature, for which a Lagrangian test particle perspective might not be readily available. One of the main motivations for this study is the understanding of fast propagation phenomena in magnetically confined plasmas. The basic problem can be understood without entering into the details concerning the plasma system. The top panel in Fig. 8 shows the basic configuration of interest, where $T_{0}$ is perturbed by a pulse at the edge of the domain. The problem then is to study the relaxation of the system back to the steady state. This type of perturbative transport experiments are commonly performed in magnetically confined fusion devices where a plasma is suddenly cooled at the edge. It has been observed in several experiments that such cold pulse perturbations travel from the edge to the center of the device at speeds significantly greater than the typical diffusive time scales. Because of this, attempts to model some of these experiments using the diffusion equation have failed. Here we discuss the use of non-local transport models as an alternative to diffusive models to describe these phenomena.

By non-local we mean that, contrary to the Fourier-Fick's local prescription in Eq. (46), the flux $q$ at a given point depends on the gradient of $T$ throughout the entire domain. The generic mathematical structure of these nonlocal models is

$q(x)=-\chi \int \mathcal{K}(x-y) \partial_{y} T(y) d y$,

where the kernel $\mathcal{K}$ determines the level of non-locality. In the case when $\mathcal{K}=\delta\left(x-x^{\prime}\right)$, Eq. (47) reduces to the familiar Fourier-Fick prescription in Eq. (46), where for simplicity we assume $V=0$.

Non-local transport is a problem of significant interest in plasma physics, see for example Callen and Kissick (1997) and references therein. Flux-gradient relations of the form in
Eq. (47) have been used in the study of parallel electron heat transport in magnetized plasmas (Held et al., 2001), and in the study of transport due to long scale-length fluctuations (Yoshizawa et al., 2003). However, the physics behind the non-local models discussed here is different, and it is based on the theory of non-Gaussian stochastic processes. Motivated by the results discussed in the previous sections, we model the non-local flux-gradient relation in Eq. (47) using fractional derivative type operators of the form

$q=-\chi(x)\left[l_{a} \mathcal{D}_{x}^{\alpha}-r_{x} \mathcal{D}_{b}^{\alpha}\right] T$,

where $\chi$ can depend on $x$ and

${ }_{a} \mathcal{D}_{x}^{\alpha} T=\frac{1}{\Gamma(2-\alpha)} \int_{a}^{x} \frac{T^{\prime}(y)-T^{\prime}(a)}{(x-y)^{\alpha-1}} d y$,

${ }_{x} \mathcal{D}_{b}^{\alpha} T=\frac{1}{\Gamma(2-\alpha)} \int_{x}^{b} \frac{T^{\prime}(b)-T^{\prime}(y)}{(y-x)^{\alpha-1}} d y$,

where $T^{\prime}=\partial_{x} T$, and $l$ and $r$ are defined in Eqs. (31). Note that the operators ${ }_{a} \mathcal{D}_{x}^{\alpha}$ and ${ }_{x} \mathcal{D}_{b}^{\alpha}$ are not exactly the usual Riemann-Liouville fractional derivative operators introduced in Eqs. (29) and (30). As discussed in del-Castillo-Negrete (2006); del-Castillo-Negrete et al. (2008) this difference has to do with the important issue that in a finite size domain $x \in(a, b)$ the Riemann-Liouville operators must be regularized to incorporate general boundary conditions of physical interest.

The role of non-locality and asymmetry in transport is illustrated Fig. 7 that shows the time evolution of a localized pulse initial condition in the model in Eqs. (45) and (48) with $\alpha=1.3$ and $\theta=0.5$. As the top panel shows, due to the asymmetry, $\theta \neq 0$, the peak of the distribution shifts to the right. It can be shown that the peak of the profile, $x_{\mathrm{m}}$, during the drift satisfies (del-Castillo-Negrete, 2006),

$x_{\mathrm{m}}(t)=\eta_{\mathrm{m}} \chi^{1 / \alpha} t^{\beta / \alpha}$,

where

$\eta_{\mathrm{m}}=\theta\left(\frac{\alpha+1}{2 \alpha}\right) \alpha^{1 / \alpha}\left|\tan \left(\frac{\alpha \pi}{2}\right)\right|$.

As expected, in the $\theta=0$ symmetric case and in the $\alpha=$ 2 diffusive limit, the drift vanishes. This drift results from the existence of "up-hill" transport which is a generic feature of non-local transport models. In the Fourier-Fick's prescription the flux dependence on the local gradient is always "down-hill", i.e., in the direction opposite to the local gradient. However, as the vertical lines in the top and middle panels of Fig. 7 indicate, in this case there is region of "uphill" transport in which the flux is in the same direction as the gradient. Moreover, as the bottom panel of Fig. 7 shows, in the non-local decay of the pulse, the flux-gradient relation is not linear like in the Fourier-Fick's diffusive case, it is in fact multivalued. The multivalued relation between $q$ and $-\partial_{x} T$ is a generic feature of non-local transport models with or without asymmetry. 
(a)

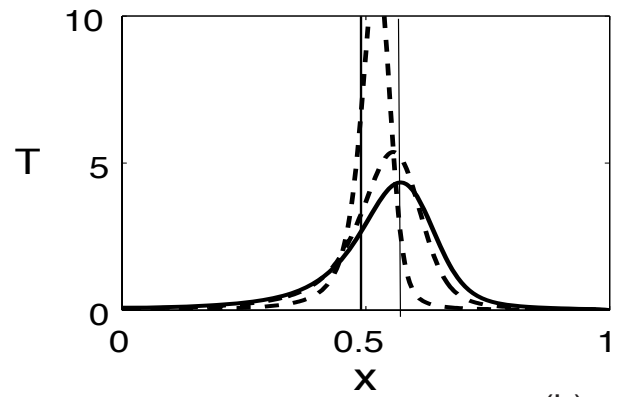

(b)

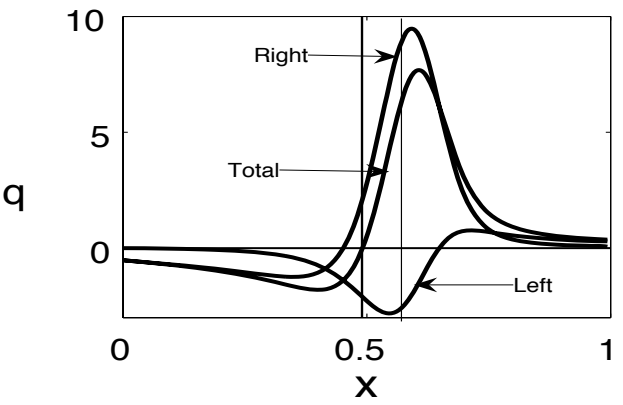

(c)

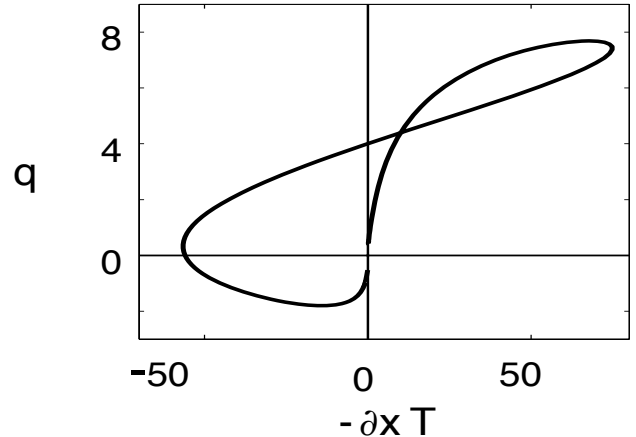

Fig. 7. Non-local transport of a localized pulse initial condition according to the fractional diffusion model in Eqs. (45) and (48) with $\alpha=1.3, \beta=1$, and $\theta=0.5$. The curve plotted with the solid line in panel (a) shows the profile at the final time, and the curves plotted with dashed lines show the profiles at earlier times. The drift of the distribution results from the asymmetry $\theta \neq 0$ of the fractional operator. Panel (b) shows the left, $q_{l}$, the right, $q_{r}$, and the total non-local flux, $q$, and panel (c) shows the flux-gradient relation. Contrary to the Fourier-Fick's linear relation, $q=-\chi \partial_{x} T$, $q$ and $-\partial_{x} T$ exhibit a nonlinear, multivalued relation. The top, left quadrant, $-\partial_{x} T<0$ and $q>0$ corresponds to up-hill transport that occurs in the region bounded by the two vertical lines in panels (a) and (b).

In the study of the propagation of pulse perturbations, the first step is the computation of the steady equilibrium temperature profile, $T_{0}(x)$ in the presence of a source of the form

$S=S_{0} \exp \left[-\frac{\left(x-\mu_{\mathrm{s}}\right)^{2}}{2 \sigma_{\mathrm{s}}^{2}}\right]$,
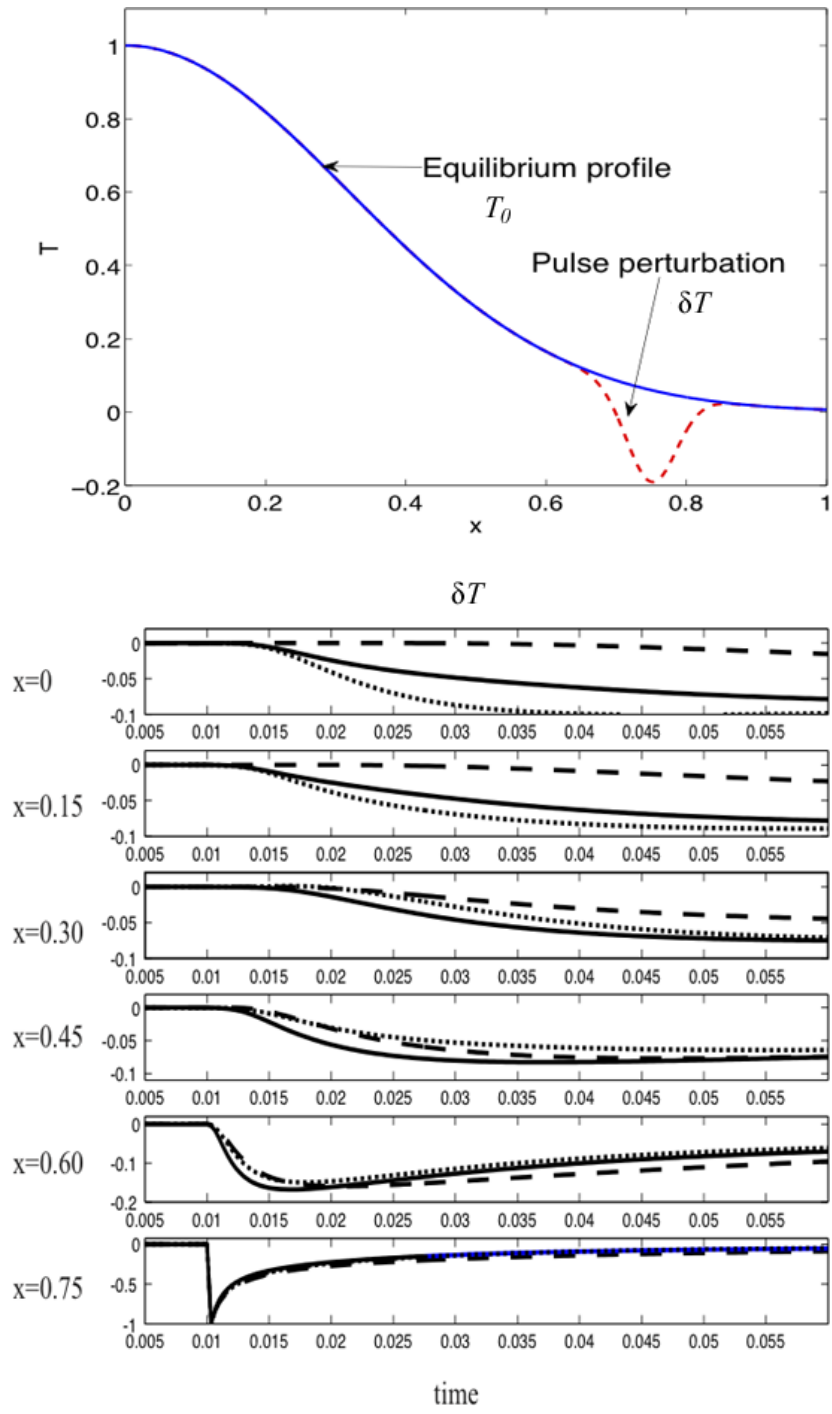

Fig. 8. Non-local fast pulse propagation. As shown in the top panel, perturbative transport simulations follow the evolution of a localized perturbation (dashed line) of an steady state passive tracer profile (solid line). The bottom panel shows the time traces of the normalized tracer perturbation, $\delta T=\delta T /|\min [\delta T(x, 0)]|$, at different locations along the $\mathrm{x}$-domain. In the local diffusive case (dashed lines) the normalized propagation speed from the edge, $x=0.75$, to the center, $x=0$, of the domain is $\hat{V}_{p}=1$. In the fractional case with $\alpha=1.75$ (solid lines), $\hat{V}_{\mathrm{p}}=6.3$, and in the fractional case with $\alpha=1.25$ (dotted line), $\hat{V}_{\mathrm{p}}=9.6$.

with $\mu_{\mathrm{s}}=0$, and $\sigma_{\mathrm{s}}=0.075$. For each simulation, the source amplitude was selected so that $T_{0}(0)=1$. The simulations followed the spatio-temporal evolution of the perturbed temperature, $\delta T(x, t)=T(x, t)-T_{0}(x)$, with initial condition

$\delta T(x, 0)=-A \exp \left[-\frac{\left(x-\mu_{\mathrm{p}}\right)^{2}}{2 \sigma_{p}^{2}}\right]$, 
where $A=0.3, \mu_{\mathrm{p}}=0.75$, and $\sigma_{\mathrm{p}}=0.03$. Details on the numerical method used to integrate the fractional transport model can be found in del-Castillo-Negrete (2006). The bottom panel in Fig. 8 shows the time evolution of the normalized tracer perturbation, $\delta \hat{T}=\delta T /|\min [\delta T(x, 0)]|$, at different locations along the $\mathrm{x}$-domain. We define the mean pulse propagation speed as the ratio of the normalized distance and the time delay, $V_{\mathrm{p}}=1 / \delta t$. The time delay is defined as the time required for the scalar field at $x=0$ to exhibit a drop of size $\delta T_{\mathrm{c}}$. That is, $\delta T(0, \delta t)=\delta T_{\mathrm{c}}$. For the value of the threshold we choose $\delta T_{\mathrm{c}}=-0.0375$. We considered three case: an $\alpha=2$ diffusive case, and two fractional cases with $\alpha=1.75$ and $\alpha=1.25$. The main conclusion is that non-locality can lead to a considerable increase of the pulse speed. In particular, the numerical results show that for the same value of $\chi, V_{\mathrm{p}}$ for $\alpha=$ 1.25 is about 10 times bigger than the diffusive speed. This idea was used in del-Castillo-Negrete et al. (2008) to model perturbative experiments on cold temperature pulse propagation in the Join European Torus (JET) magnetically confined controlled fusion device.

To conclude we present recent results on the role of non-locality in the propagation of pulses through transport barriers. The local and non-local diffusivities are assumed to be of the form

$\chi_{d}=\chi_{d 0}-\zeta e^{-\left(x-x_{0}\right)^{2} / w}$,

and

$$
\begin{aligned}
\chi_{\mathrm{nl}}= & \frac{\chi_{\mathrm{nl} 0}}{2}\left[\tanh \left(\frac{x-x_{\mathrm{c}}}{L}\right)+\tanh \left(\frac{x_{\mathrm{c}}}{L}\right)\right] \\
& -\zeta e^{-\left(x-x_{0}\right)^{2} / w} .
\end{aligned}
$$

The tanh profile in $\chi_{\mathrm{nl}}$ is introduced to guarantee the vanishing of the non-local flux in the core region where transport is assumed to be dominated by diffusive processes. The transport barrier is modeled by introducing a dip, $e^{-\left(x-x_{0}\right)^{2} / w}$, in the diffusivity profiles. In the calculations reported here $\chi_{d 0}=1, x_{0}=0.5, \zeta=0.95, \chi_{\mathrm{n} 10}=1, x_{\mathrm{c}}=0.1$, $L=0.025$, and $w=0.005$. In the non-local simulations, $\alpha=1.25$. Figure 9 shows the spatio-temporal evolution of $\delta T$. The top panel shows the case of diffusive transport, $\chi_{\mathrm{n} 10}=0$, in the absence of transport barriers. In this case, the pulse spreads throughout the plasma domain in a slow, diffusive time scale. As expected, as shown in the middle panel, in the presence of a transport barrier the diffusive propagation of the pulse is stopped. However, in the presence of non-local transport the pulse dynamics is fundamentally different. As the bottom panel in Fig. 9 shows, in this case the pulse can in fact go through the transport barrier. This "tunneling" effect is a unique novel property of non-local transport.
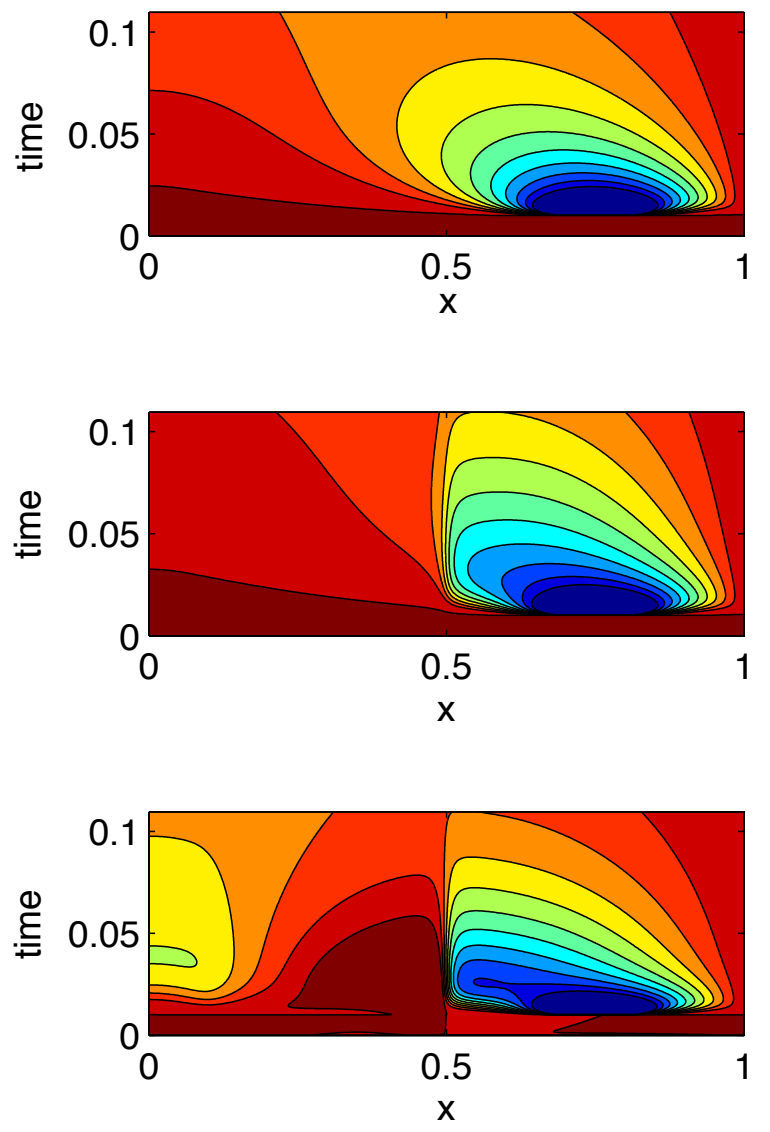

Fig. 9. Non-local "tunneling" of perturbations across a transport barrier. The figure shows the space-time evolution of the normalized passive tracer perturbation $\delta \hat{T}=\delta T /|\min [\delta T(x, 0)]|$ with dark blue (red) denoting $\delta \hat{T}=1(\delta \hat{T}=0)$. The top panel corresponds to diffusive transport in the absence of transport barriers. The middle and bottom panels correspond to diffusive and non-local transport respectively in the presence of a transport barrier located at $x=0.5$.

\section{Conclusions}

We have presented a review of recent results on nondiffusive transport in fluids and plasmas. The approach was based on the study of the Lagrangian statistics of large ensembles of particles. In general, the stochasticity in the Lagrangian trajectories can result from deterministic chaos or from turbulence. The examples discussed encompass both possibilities. In the studies of transport by Rossby waves in quasigeostrophic zonal flows, the advection velocity was a smooth deterministic function but the Lagrangian trajectories exhibited Hamiltonian chaos. On the other hand, in the $\mathbf{E} \times \mathbf{B}$ transport plasma problem, the advection velocity was a non-deterministic random function obtained from the solution of a turbulence model. The main object of study was the probability density function (PDF) of individual particle displacements, also know as the propagator. Both, the fluid chaotic transport problem and the plasma turbulent 
transport problem, exhibited strongly non-Gaussian spatiotemporal self-similar PDFs. In addition, the Lagrangian statistics in both cases exhibited super-diffusive scaling, $<$ $x^{2}>\sim t^{\gamma}$ with $\gamma>1$. The modeling of these PDFs using advection-diffusion equations is out of the question because the effective diffusivity diverges, and the propagators have non-Gaussian decaying tails. The observed non-Gaussian statistics in the examples discussed has its origin on the combination of anomalously large particle displacements, known as "Levy flights", and the trapping effects of coherent structures like fluid vortices and $\mathbf{E} \times \mathbf{B}$ plasma eddies.

We have shown that the PDFs of particle displacements can be modeled using fractional diffusion equations in which regular derivatives are replaced by fractional derivatives. Fractional derivatives are integro-differential operators that provide a powerful, elegant framework to incorporate nonGaussian and non-Markovian effects on transport models. These operators naturally appear in the continuum limit of generalized random walk models that extend the Brownian motion by allowing non-Gaussian jump distribution functions and general waiting time distribution functions.

Going beyond the study of non-Gaussian Lagrangian statistics, we discussed the application of fractional derivatives to model non-local transport. The cornerstone of the diffusive transport paradigm is the Fourier-Fick's prescription according to which the flux at a given point depends only of the gradient of the transported field at that point. On the other hand, in the case of non-local transport, the flux can depend on the gradient throughout the entire domain. Although in many cases transport problems follow the Fourier-Fick's prescription, there are important situations in which this is not the case. A clear example is the fast propagation phenomena observed in perturbative transport experiments in magnetically confined plasma fusion devices. Motivated by the successful use of fractional derivatives in the study of non-diffusive Lagrangian transport, we used these operators to construct non-local models of passive scalar transport. We presented numerical results illustrating important non-local transport phenomenology including: uphill transport, multivalued flux-gradient relations, fast pulse propagation phenomena, and "tunneling" of perturbations across transport barriers.

Some of the results presented here pertain specific systems, i.e., Rossby waves in zonal flows and pressuregradient-driven plasma turbulence. However, it is important to realize that the observed non-diffusive phenomenology depends on very general non-Gaussian statistical properties and not on specific details. In particular, other systems with coherent structures and/or strong spatio-temporal correlations are likely to exhibit similar non-diffusive and non-local transport dynamics. For example, there is observational evidence of superdiffusive transport of electrons accelerated at interplanetary shocks at corotating interaction regions (CIRs) (Perri and Zimbardo, 2007) and observational evidence of ion superdiffusion at the solar wind termination shock (Perri and Zimbardo, 2009). It would be interesting to explore the application of the ideas and techniques discussed here to these systems. On the other hand, knowing the key role that coherent structures play in non-diffusive behavior, dynamical systems tools used to identify Lagrangian coherent structures (LCS) in fluid turbulence (Haller and Yuan, 2000; Mathur et al., 2007) and plasma turbulence (Padberg et al., 2007) should be of relevance to the transport studies discussed in the present paper.

Acknowledgements. This work was sponsored by the Oak Ridge National Laboratory, managed by UT-Battelle, LLC, for the U.S. Department of Energy under contract DE-AC05-00OR22725.

Edited by: B. Tsurutani

Reviewed by: two anonymous referees

\section{References}

Bouchaud, J. P. and Georges, A.: Anomalous diffusion in disordered media: Statistical mechanisms, models and physical applications, Phys. Rep., 195, p. 127, doi:10.1016/03701573(90)90099-N, 1990.

Callen, J. D. and Kissick, M. W.: Evidence and concepts for non-local transport, Plasma Phys. Contr. F., 39, B173, doi:10.1088/0741-3335/39/12B/014, 1997.

Carreras, B. A., Garcia, L., and Diamond, P. H.: Theory of resistive pressure-gradient-driven turbulence, Phys. Fluids, 30, 1388, doi:10.1063/1.866518, 1987.

Cartea, A. and del-Castillo-Negrete, D.: Fluid limit of the continuous-time random walk with general Levy jump distribution functions, Phys. Rev. E, 76, 041105, doi:10.1103/PhysRevE.76.041105, 2007.

del-Castillo-Negrete, D.: Asymmetric transport and non-Gaussian statistics of passive scalars in vortices in shear, Phys. Fluids, 10, p. 576, 1998 .

del-Castillo-Negrete, D.: Chaotic transport in zonal flows in analogous fluid and plasma systems, Phys. Plasmas, 7, 1702, doi:10.1063/1.873988, 2000.

del-Castillo-Negrete, D.: Fractional diffusion models of nonlocal transport, Phys. Plasmas, 13, 082308, doi:10.1063/1.2336114, 2006.

del-Castillo-Negrete, D. and Morrison, P. J.: Chaotic Transport by Rossby Waves in Shear Flow, Phys. Fluids A, 5, 948, doi:10.1063/1.858639, 1993.

del-Castillo-Negrete, D., Carreras, B. A., and Lynch, V. E.: Fractional diffusion in plasma turbulence, Phys. Plasmas, 11, 3854, doi:10.1063/1.1767097, 2004.

del-Castillo-Negrete, D., Carreras, B. A., and Lynch, V. E.: Non-diffusive transport in plasma turbulence: a fractional diffusion approach, Phys. Rev. Lett., 94, 065003, doi:10.1103/PhysRevLett.94.065003, 2005.

del-Castillo-Negrete, D., Mantica, P., Naulin, V., and Rasmussen, J.: Fractional diffusion models of non-local perturbative transport: numerical results and applications to JET experiments, 
Nucl. Fusion, 48, 075009, doi:10.1088/0029-5515/48/7/075009, 2008.

Gustafson, K., del-Castillo-Negrete, D., and Dorland, W.: Finite Larmor radius effects on non-diffusive tracer transport in a zonal flow, Phys. Plasmas, 15, 102309, doi:10.1063/1.3003072, 2008.

Haller, G. and Yuan, G.: Lagrangian coherent structures and mixing in two-dimensional turbulence, Physica D, 147, 352-370, 2000.

Held, E. D., Callen, J. D., Hegna, C. C., and Sovinec, C. R.: Conductive Electron Heat Flow along Magnetic Field Lines, Phys. Plasmas, 8, 1171, doi:10.1063/1.1349876, 2001.

Horton, W. and Hasegawa, A.: Quasi-two-dimensional dynamics of plasmas and fluids, Chaos, 4, p. 227, 1994.

Horton, W. and Ichikawa, Y. H.: Chaos and structures in nonlinear plasmas, World Scientific Publishing, 1996.

Mainardi, F., Luchko, Y., and Pagnini, G.: The fundamental solution of the space-time fractional diffusion equation, Fractional Calculus and Applied Analysis, 4, 153-192, 2001.

Mathur, M., Haller, G., Peacock, T., Ruppert-Felsot, J. E., and Swinney, H. L.: Uncovering the Lagrangian Skeleton of Turbulence, Phys. Rev. Lett. 98, 144502, doi:10.1103/PhysRevLett.98.144502, 2007.

Metzler, R. and Klafter, J.: The random walk's guide to anomalous diffusion: A fractional dynamics approach, Phys. Rep., 339, 177, 2000.

Montroll, E. W. and Weiss, G. H.: Random walks on lattices, J. Math. Phys., 6, p. 167, 1965.

Montroll, E. W. and Shlesinger, M. F.: , in: Nonequilibrium Phenomena, II. From Stochastics to Hydrodynamics, edited by: Lebowitz, J. L. and Montroll, E. W., Elsevier Science Publishers BV, 1984.

Nicholson, D.: Introduction to Plasma Theory, Wiley, 1983.

Padberg, K., Hauff, T., Jenko, F., and Junge, O.: Lagrangian structures and transport in turbulent magnetized plasmas, New J. Phys., 9, 400 pp., 2007.

Paul, W. and Baschnagel, J.: Stochastic Processes: From Physics to Finance, Springer Berlin Heidelberg, 1999.

Perri, S. and Zimbardo, G.: Evidence of Superdiffusive Transport of Electrons Accelerated at Interplanetary Shocks, Astrophys. J. Lett., 671, L177, doi:10.1086/525523, 2007.

Perri, S. and Zimbardo, G.: Ion Superdiffusion at the Solar Wind Termination Shock, Astrophys. J. Lett., 693, L118, doi:10.1088/0004-637X/693/2/L118, 2009.
Ottino, J. M.: The kinematics of mixing-stretching, chaos, and transport, Cambridge University Press, 1989.

Pedlosky, J.: Geophysical fluid dynamics, 2nd edn., SpringerVerlag, New York, 1987.

Petviashvili, V. I. and Pokhotelov, O. A.: Solitary Waves in Plasmas and in the Atmosphere, Gordon and Breach, PA, 1992.

Podlubny, I.: Fractional Differential Equations, Academic Press, San Diego, 1999.

Saichev, A. and Zaslavsky, G. M.: Fractional kinetic equations: Solutions and applications, Chaos, 7(4), 753 pp., 1997.

Samko, S. G., Kilbas, A. A., and Marichev, O. I.: Fractional Integrals and Derivatives, Gordon and Breach Science Publishers, Amsterdam, 1993.

Sanchez, R., Carreras, B. A., Newman, D. E., Lynch, V. E., and van Milligen, B. Ph.: Renormalization of tracer turbulence leading to fractional differential equations, Phys. Rev. E, 74, 016305, doi:10.1103/PhysRevE.74.016305, 2006.

Sanchez, R., Newman, D. E., Leboeuf, J. N., Decyk, V. K., and Carreras, B. A.: Nature of transport across sheared zonal flows in electrostatic ion-temperature-gradient gyrokinetic plasma turbulence, Phys. Rev. Lett., 101, 205002, doi:10.1103/PhysRevLett.101.205002, 2008.

Scalas, E., Gorenflo, R., and Mainardi, F.: Uncoupled continuous-time random walks: Solution and limiting behavior of the master equation, Phys. Rev. E, 69, 011107, doi:10.1103/PhysRevE.69.011107, 2004.

Solomon, T. H., Weeks, E. R., and Swinney, H. L.: Observations of anomalous diffusion and Levy flights in a 2-dimensional rotating flow, Phys. Rev. Lett., 71, 3975, doi:10.1103/PhysRevLett.71.3975, 1993.

Spizzo, G., White, R. B., Cappello, S., and Marrelli, L.: Nonlocal transport in the reversed field pinch, Plasma Phys. Contr. F., 51, 124026, doi:10.1088/0741-3335/51/12/124026, 2009.

Tabor, M.: Chaos and Integrability in Nonlinear Dynamics, Wiley, John \& Sons, Incorporated, New York, 1989.

Yoshizawa, A., Itoh, S. I., and Itoh, K.: Plasma and Fluid Turbulence, Institute of Physics, Series in Plasma Physics, 2003.

van Milligen, B. Ph., Sanchez, R., and Carreras, B. A.: Probabilistic finite-size transport models for fusion: Anomalous transport and scaling laws, Phys. Plasmas, 11, 2272, doi:10.1063/1.1701893, 2004. 